\title{
Synthesis of Spiropyrrolidines via 1, 3 Anionic Cycloaddition
}

\author{
Abdul Wahab J. Al-Hamdany Abdul Majeed M. Dabbagh \\ Omar A. Shareef \\ Department of Chemistry \\ College of Science \\ University of Mosul
}

(Received 11/10/2011;Accepted 21/11/2011)

\begin{abstract}
In this work, series of substituted $\alpha, \beta$-unsaturated carbonyl compounds (A1-8) had been prepared using Claisen-Schmidt condensation. Also, series of substituted Schiff bases (N-arylidene-4-picolyamine) (B1-6) were prepared by the condensation of 4-picolyamine with various aromatic aldehydes.

The reaction of the above mentioned materials $(\mathrm{A}+\mathrm{B})$ afforded the desired products [Spiropyrrolidines (S1-15)]. The reaction occurred through the formation of azallyl anion which acted as a nucleophile that has the ability to attack the exocylic double bond of chalcones through the 1,3-anionic cycloaddition mechanism to form the product.

The structure for all synthesized compounds (chalcones, Schiff bases, pyrrolidines) had been confirmed by the spectral data obtained by (UV, IR, ${ }^{1} \mathrm{H}-\mathrm{NMR}$ ) as well as the determination of physical properties.
\end{abstract}

Keywords: spiropyrrolidine, 1,3-anionic cycloaddition.

\section{تحضير بهض مركبلت السبليرومليرولينينمن خلال الضالفة 1، 3 الانيونية}

\section{الملغص}

م في هذا البهث تحضيرسلسلة من معوضلت مركبلت الكاربونيل الفا-بيتا- غير المشبعة (8 1- A

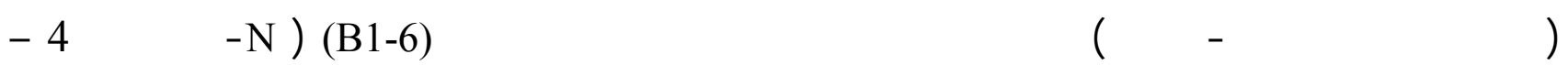
بيكولايل لمين) بمعوضلت مختلفة من خلال تكاق 4 -بيكوليل لمين مع الديهايدات اروماتية ذات معوضات مختلفة.

كما مة إجراء قفاعل بين المواد المنكورة أعلاه (A , B) للحصول على المركبلت الحلقية

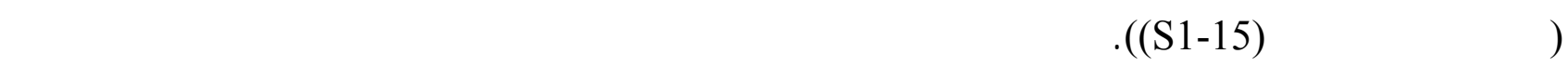
نيوكليوفيل له القابلية على مهلجمة الآصرة المزدوجة (C=C) للجالكونات، وذلك عبر ميكانيكية الإضافة الحقية الانيونية نوع (1،3) مُ إثبات الترلكيب للمواد المحضرة ( الجالكونات، قواعدشيف، وكذلك 
البايروليينات) بلستخدلم الطرائق الطيفية (UV, IR , ,H-NMR) فضلا عن اليجاد بعض الصفلت الفيزياوية

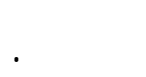

الهامل الدالة: سبايروبايروليين، 1، 3 الانيونية.

\section{INTRODUCTION}

Spiro compounds having cyclic structure fused at a central carbon are of recent interest due to their interesting structural implications on biological system (Jayashankaran et al., 2005). The asymmetric characteristics of the molecule due to the chiral spiro carbon is one of the important criteria of the biological activities (Ghandi et al., 2009).

Spiro compounds represent an important class of naturally occurring substances characterized by highly pronounced biological properties (Raj and Raghnathan, 2005).

The most developed route for the synthesis of these compounds depends on the cycloaddition to an exocyclic bond. (Poornachandran and Raghunathan, 2010) (Fisera et al., 1994).

The 1,3-Anionic cycloaddition provides a way for the synthesis of many spiroheterocycles through the cycloaddition reaction of nonstabilised azomethine ylides with the Chalcones (Waldmann, 1995). Also highly substituted pyrrolidines have attracted much interest as they contribute the central structural element of many alkaloids and pharmacological active compounds (Luibineau et al., 1995) (Deshong and Leginus, 1983) (Manikandan et al., 2001) (Subramaniyan and Raghunathan, 2001) on the synthesis of novel spiropyrrolidinyl derivatives we have examined the 1,3-Anionic cycloaddition reaction of E-2-arylidene-1-tetralones with the azomethine ylide generated by treatment the Schiff bases with sodium hydroxide.

\section{EXPERIMENTAL}

Melting points were determined by Electrothermal 9300 Engineering LTD Apparatus (the melting points are uncorrected), the boiling points were determined by inverted capillary in a thiele tube using paraffin colorless oil (Shriner et al., 1964). A shimadzu UVVisible spectrophotometer UV-1650/PC connected to a computer with pentium 4 processor Quartz cells $(1 \times 1 \times 3 \mathrm{~cm})$ were used to for UV measurements. Fourier - Transform Infrared (FT-IR) spectrophotometer, Tensor Co. Brucker, 2003 Germany, was used to run IR spectra. The nuclear magnetic resonance $\left({ }^{1} \mathrm{H}-\mathrm{NMR}\right)$ spectra were performed using $\left(300 \mathrm{MH}_{\mathrm{z}}\right)$ Brucker, tetramethyl silane (TMS) as an internal standard, and $\mathrm{CDCl}_{3}$ as a solvent in Al Al-Bayt University, Jordan.

\section{Preparation of chalcones ( arylidenecycloalkanone and derivatives (A1-8)}

A solution of $2.2 \mathrm{~g}$ of sodium hydroxide in $20 \mathrm{ml}$ of water and $12.25 \mathrm{ml}$ of ethanol were placed in a $50 \mathrm{ml}$ round -bottomed flask provided with a mechanical stirrer. The flask is immersed in a bath of crushed ice. A (0.0215) mol of freshly distilled cycloalkanone was added. Stirring is then started and 0.043 mole of pure aldehyde was added. The temperature of the mixture is kept at about $25^{\circ} \mathrm{C}$ (the limits are $15-30{ }^{\circ} \mathrm{C}$ ) with vigorous stirring until 
turns to a very thick consistency. The stirrer was stopped and the reaction mixture was left in an ice crushed bath or refrigerator overnight. The product was filtered using Buchner funnel or a sintered glass funnel, washed with cold water until the washings were neutral to litmus, and then with $20 \mathrm{ml}$ of ice -cold ethanol. After drying in air, the crude chalcone was recrystallized from ethanol. The product was always handled with care as it caused a skin irritation (Vogel, 1989). The physical properties of synthesized compounds and spectral data illustrated in Tables 1 and 2. The melting points, infrared and ultraviolet data, were in a good agreement with the reported values. (Parikh, 1974).

\section{Preparation of Schiff bases [N-Arylidene 4-picolyamine] (B1-B6)}

In a $100 \mathrm{ml}$ beaker, 0.01 mole of (4-picolyl amine) was heated with 0.01 mole of substituted benzaldehyde and $10 \mathrm{ml}$ of n-butanol for 10 minutes at $\left(100^{\circ} \mathrm{C}\right)$. The reaction mixture was then cooled and filtered. The precipitate was dried and recrystallized from ethanol (the liquid products were purified by distillation). The physical properties and spectral data of Schiff bases (B1-6) illustrated in Table 3 and 4 respectevely. The Infrared and ultraviolet data were in a good agreement with the reported values. (Bin et al., 2009).

Table 1: Names and some physical properties of substituted chalcones A (1-8).

\begin{tabular}{|c|c|c|c|c|c|c|}
\hline $\begin{array}{c}\text { Compd. } \\
\text { No. }\end{array}$ & $\mathbf{Y}$ & Ar & $\begin{array}{c}\text { Reaction } \\
\text { time /h }\end{array}$ & Color & m.p., ${ }^{\circ} \mathrm{C}$ & $\begin{array}{c}\text { Yield } \\
\%\end{array}$ \\
\hline A1 & $\mathrm{H}$ & 2-furfuryl & 3 & Yellow & $71-73$ & 75 \\
\hline $\mathrm{A} 2$ & 6-Meo & phenyl & 3 & Yellow & $95-97$ & 65 \\
\hline A3 & 6-Meo & 4-anisyl & 4 & Yellow & $141-143$ & 61 \\
\hline A4 & $6-\mathrm{OCH}_{3}$ & p-nitro phenyl & 3 & Yellow & $98-101$ & 58 \\
\hline A5 & $6-\mathrm{OCH}_{3}$ & p-chloro phenyl & 4 & White & $91-93$ & 60 \\
\hline A6 & $\mathrm{H}$ & phenyl & 4.5 & Yellow & $105-107$ & 80 \\
\hline A7 & $\mathrm{H}$ & 4-anisyl & 3 & Yellow & 109-111 & 60 \\
\hline A8 & $\mathrm{H}$ & p-chloro phenyl & 3 & White & $72-75$ & 83 \\
\hline
\end{tabular}

Table 2: Spectral data of Chalcones (A1-8).

\begin{tabular}{|c|c|c|c|c|c|c|}
\hline \multirow{2}{*}{$\begin{array}{c}\text { Compd. } \\
\text { No. }\end{array}$} & \multicolumn{4}{|c|}{ IR (KBr), $\mathrm{c} \mathrm{cm}^{-1}$} & & \multirow{2}{*}{$\begin{array}{c}\mathrm{U.V} . \\
(\mathrm{CHCl}) \\
\lambda_{\max }(\mathrm{nm})\end{array}$} \\
\hline & $\mathrm{C}=\mathrm{O}$ & $\mathrm{C}=\mathrm{C}$ & $C=-C$ & C-O-C & $\mathrm{N} \quad \ldots \mathrm{O}$ & \\
\hline $\mathrm{A} 1$ & 1664 & 1620 & 1591 & 1233 & & 352 \\
\hline $\mathrm{A} 2$ & 1604 & 1660 & 1586 & ----- & & 324 \\
\hline A3 & 1663 & 1593 & 1508 & ----- & & 338 \\
\hline A4 & 1681 & 1639 & 1506 & ----- & $\begin{array}{l}\text { 1333 Sym } \\
\text { 1549A sym }\end{array}$ & 390 \\
\hline A5 & 1656 & 1606 & 1590 & 1226 & & 304 \\
\hline A6 & 1660 & 1624 & 1589 & ------ & & 310 \\
\hline A7 & 1666 & 1600 & 1508 & 1222 & & 342 \\
\hline A8 & 1662 & 1604 & 1514 & $-\ldots$ & & 386 \\
\hline
\end{tabular}


Table 3: Physical properties of the Schiff bases (B1-6).

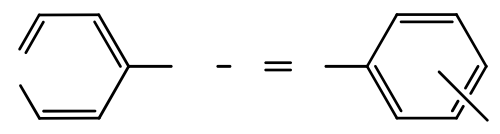

\begin{tabular}{|c|c|c|c|c|}
\hline $\begin{array}{c}\text { Compd. } \\
\text { No. }\end{array}$ & $\mathrm{X}$ & Color \& phase & m.p. or b.P /C & $\begin{array}{c}\text { Yield } \\
\mathbf{\%}\end{array}$ \\
\hline B1 & $\mathrm{H}$ & Yellow precipitate & $70-72$ & 76 \\
\hline B2 & $4-\mathrm{OCH}_{3}$ & Orange liquid & $205-208$ & 62 \\
\hline B3 & $4-\mathrm{NO}_{2}$ & Brown liquid & $130-132$ & 73 \\
\hline B4 & $4-\mathrm{Cl}$ & Pale yellow precipitate & $62-64$ & 69 \\
\hline B5 & $3,4-\mathrm{di4}_{2} \mathrm{OCH}_{3}$ & Orange liquid & $143-146$ & 60 \\
\hline B6 & $4-\mathrm{CH}_{3}$ & White precipitate & $40-43$ & 75 \\
\hline
\end{tabular}

Table 4 : Spectral data of the Schiff bases (B1-6).

\begin{tabular}{|c|c|c|c|c|c|c|}
\hline \multirow{2}{*}{$\begin{array}{c}\text { Compd. } \\
\text { No. }\end{array}$} & \multicolumn{5}{|c|}{ IR(KBr), v(cm $\left.{ }^{-1}\right)$} & \multirow{2}{*}{$\begin{array}{c}\mathbf{U} \cdot \mathbf{V} \\
\lambda_{\max } / \mathrm{nm}\end{array}$} \\
\hline & $\mathbf{C}=\mathbf{N}$ & C-O-C & $\begin{array}{c}\text { Asym } \\
\mathrm{N}=--0\end{array}$ & $\underset{-}{\text { Sym }}$ & C-Cl & \\
\hline B1 & 1594 & ---- & ----- & ----- & ----- & 280 \\
\hline B2 & 1606 & 1251 & ----- & ----- & ----- & 288 \\
\hline B3 & 1600 & $\begin{array}{l}---- \\
\end{array}$ & 1519 & 1342 & ----- & 285 \\
\hline B4 & 1633 & ----- & ----- & ----- & 752 & 292 \\
\hline B5 & 1602 & 1251 & ----- & $\begin{array}{l}---- \\
\end{array}$ & $\begin{array}{l}---- \\
\end{array}$ & 284 \\
\hline B6 & 1606 & ----- & $\begin{array}{ll}---- \\
\end{array}$ & $\begin{array}{l}---- \\
\end{array}$ & $\begin{array}{l}---- \\
\end{array}$ & 282 \\
\hline
\end{tabular}

\section{Condensation of chalcones with Schiff bases}

A mixture of Schiff bases $(0.01$ mole $)$ and chalcone $(0.01$ mole $)$ was magnetically stirred in the presence of $(3 \mathrm{ml})$ of $(0.01 \mathrm{~mole})$ alcoholic sodium hydroxide solution (Popandova et al., 1989). The mixture was allowed to stand overnight, water (100 ml) was then added to the reaction mixture. The separated precipitate was washed with water until the filtrate became clear and neutral. The solid product was then dried and recrystallized from methanol-ethyl acetate (8:2). The physical properties and the spectral data were listed in Table (5 and 6) respectively. 
Table 5: Physical properties of Spiropyrrolidines (S1-15)

\begin{tabular}{|c|c|c|c|c|c|}
\hline $\begin{array}{c}\text { Compd. } \\
\text { No. }\end{array}$ & Ar & $\mathbf{X}$ & Color & $\mathbf{m} \cdot \mathbf{p} / \mathbf{C}$ & Yield \% \\
\hline S1 & 2-furfuryl & $\mathrm{H}$ & Pale orange & $167-171$ & 51 \\
\hline S2 & 2-furfuryl & $\mathrm{H}$ & Pale yellow & $15-139$ & 45 \\
\hline S3 & 2-furfuryl & $4-\mathrm{OCH}_{3}$ & Pale red & $110-115$ & 56 \\
\hline S4 & Phenyl & $\mathrm{H}$ & Brown & $198-204$ & 50 \\
\hline S5 & Phenyl & $4-\mathrm{Cl}$ & Yellowish brown & $149-153$ & 56 \\
\hline S6 & Phenyl & $4-\mathrm{OCH}_{3}$ & Brown & $132-136$ & 58 \\
\hline $\mathrm{S} 7$ & 4-anisyl & $\mathrm{H}$ & Pale brown & $121-125$ & 38 \\
\hline S8 & Phenyl & $4-\mathrm{NO}_{2}$ & Dark brown & $220-225$ & 41 \\
\hline S9 & 4-Chloro phenyl & $4-\mathrm{OCH}_{3}$ & Dark orange & $155-158$ & 43 \\
\hline $\mathrm{S} 10$ & 4-Chloro phenyl & $\mathrm{H}$ & Pale brown & $141-144$ & 45 \\
\hline $\mathrm{S} 11$ & 4-anisyl & $4-\mathrm{Cl}$ & Dark brown & $110-113$ & 36 \\
\hline $\mathrm{S} 12$ & 4-anisyl & $4-\mathrm{NO}_{2}$ & Pale red & $135-139$ & 45 \\
\hline $\mathrm{S} 13$ & $4-\mathrm{Cl}$ & $4-\mathrm{NO}_{2}$ & Dark brown & $160-164$ & 57 \\
\hline $\mathrm{S} 14$ & Phenyl & 3,4- $\mathrm{diOCH}_{3}$ & Pale yellow & $176-180$ & 47 \\
\hline S15 & Phenyl & $4-\mathrm{CH}_{3}$ & Pale brown & $184-187$ & 50 \\
\hline
\end{tabular}

\section{RESULTS AND DISCUSSION}

$\alpha-\beta$ Unsaturated carbonyl compounds are very attractive starting materials. They are considered as precursors to build other important compounds (March, 1992) such as compounds (Katritzky, 1997) through their reaction with other different compounds.

In this paper, some chalcones ATs and FTs are synthesized by condensation reaction of $\alpha-\beta$ unsaturated ketones such as:<smiles>C=C1/C(=C/c2ccc(C)cc2)CCc2cc(C)ccc21</smiles>

Substituted-2(arylidene)-1-tetralone(AT)<smiles>C=C1/C(=C/c2cccs2)CCc2cc(C)ccc21</smiles>

Substituted-2( $\alpha$-furfurylidene)-1-tetralone(FT)

The chalcones (ATs and FTs) (A1-8) were then condensed with different Schiff bases (B1-6) of the formula

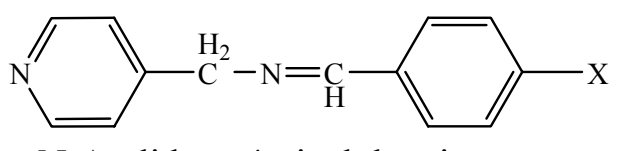

$\mathrm{N}$-Arylidene-4-picolyl amine 
To afford the corresponding substituted spiropyrrolidines (S1-15). The prepared unsaturated ketones and Schiff bases were confirmed by spectroscopic means. The physical properties and the spectral data were in quite good agreement with the published values (Parich, 1974), Table (6 and 7).

The Schemes ( $1 \mathrm{a}$ and $\mathrm{b}$ ) show the synthetic rout of compounds A,B and $\mathrm{S}$<smiles>C=C1/C(=C/c2cccs2)CCc2ccccc21</smiles><smiles>Cc1ccc([I-]c2ccccc2)cc1</smiles>

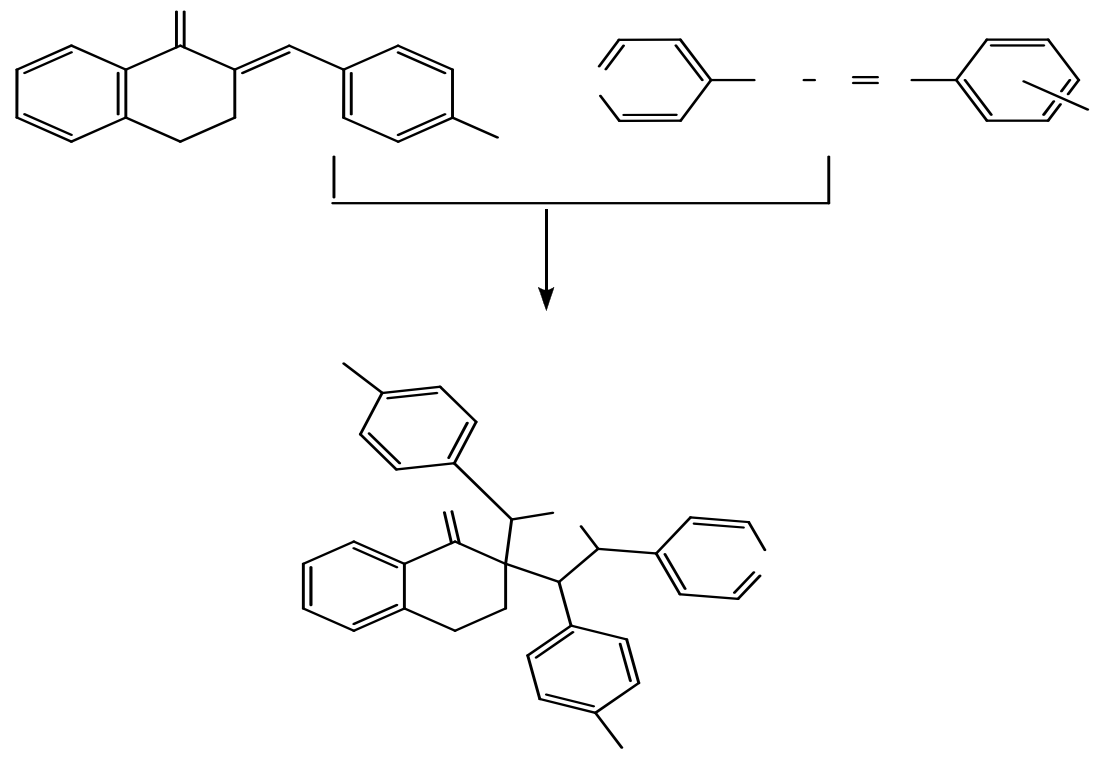




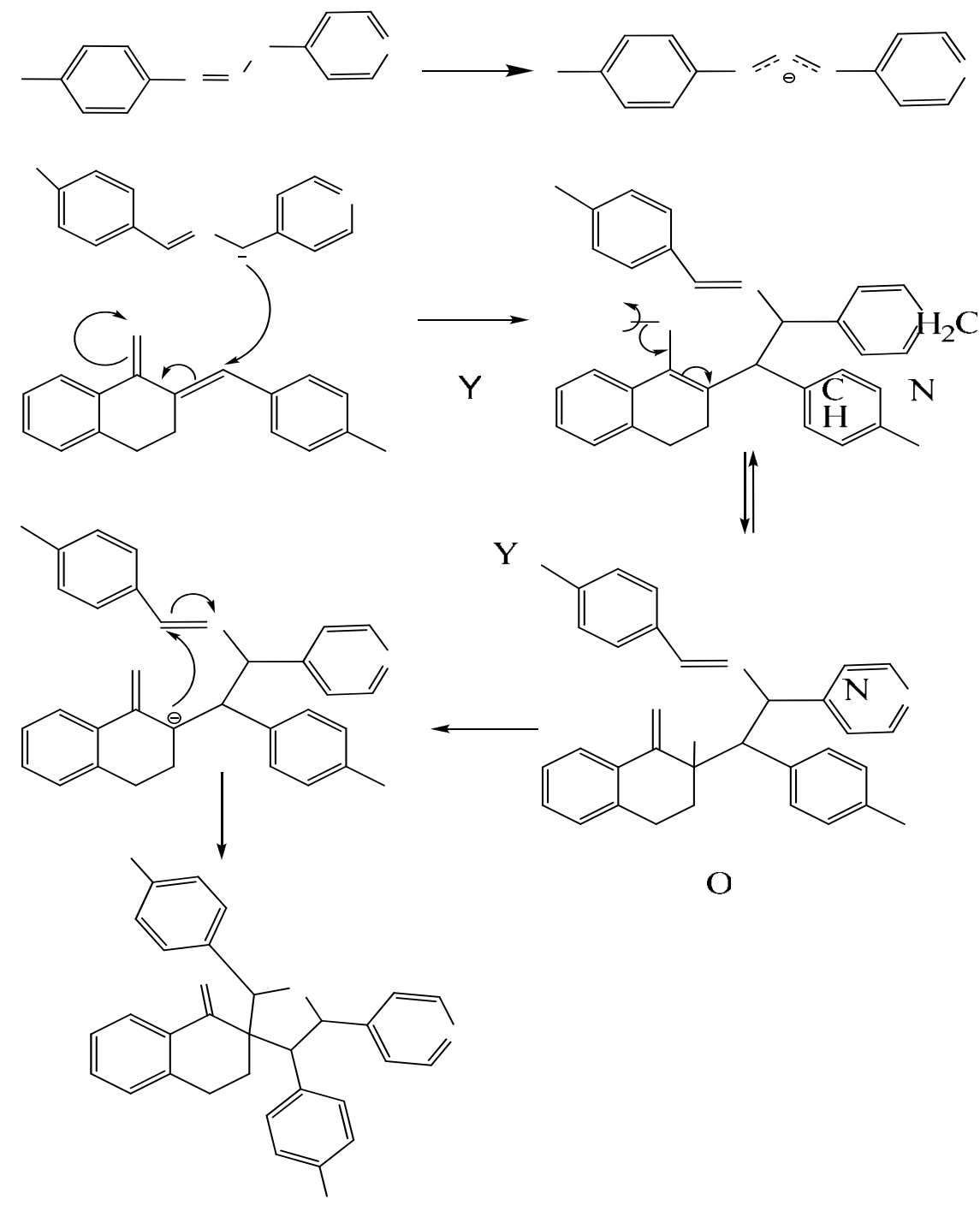

Scheme (2): Suggested Mechanism for the formation of spiropyrrolidine compounds

The structures of the synthesized compounds (or spiropyrrolidens) have been confirmed by the spectral methods. $\mathrm{N}$

The ${ }^{1} \mathrm{H}-\mathrm{NMR}$ spectrum of compound A6 is taken as a representative sample for the discussion which showed a triplet signal appeared at $\delta 2.0$ ppm for two protons attached to $\mathrm{N}$ $\left(\mathrm{C}_{1}\right)$, another triplet signal appeared at $\delta 2.2 \mathrm{ppm}$ for two protons attached to $\left(\mathrm{C}_{2}\right)$, a singlet significant band for the proton attached to (C3) showed at $\delta 6.3 \mathrm{ppm}$, while the aromatic protons showed a multiple bands at the range $\delta(6.4-7.2)$ ppm (Table 6).

The (FT-IR) spectra of compounds (A1-8) bands showed an absorption in the range of (1721-1656) $\mathrm{cm}^{-1}$ which is related to the stretching vibration of the carbonyl group, while the absorption bands in the range of $(1660-1593) \mathrm{cm}^{-1}$ related to thestretching vibration of $(\mathrm{C}=\mathrm{C})$ (double bond) (Table2). 
Table 6: ${ }^{1} \mathrm{H}-\mathrm{NMR}$ spectra data of 2-Arylidene-1-tetralones

\begin{tabular}{|c|c|c|c|c|c|}
\hline \multirow{2}{*}{$\begin{array}{c}\text { Compd. } \\
\text { No. }\end{array}$} & \multicolumn{5}{|c|}{${ }^{1} \mathrm{H}-\mathrm{NMR}\left(\mathrm{CDCl}_{3}\right) \delta \mathrm{ppm}$} \\
\hline & $\mathrm{C}_{1}-\underline{\mathrm{H}}$ & $\mathrm{C}_{2}-\underline{\mathrm{H}}$ & $\mathrm{C}_{3}-\underline{\mathrm{H}}$ & $\left(\mathrm{OCH}_{3}\right)$ & Ar- $\underline{\mathbf{H}}$ \\
\hline A1 & $2.0(\mathrm{t})(2 \mathrm{H})$ & $2.4(\mathrm{t})(2 \mathrm{H})$ & $6.3(\mathrm{~S})(1 \mathrm{H})$ & $\begin{array}{ll}---- \\
\end{array}$ & $5.5-7.1(\mathrm{~m})(7 \mathrm{H})$ \\
\hline A6 & $2.0(\mathrm{t})(2 \mathrm{H})$ & $2.2(\mathrm{t})(2 \mathrm{H})$ & $6.3(\mathrm{~S})(1 \mathrm{H})$ & $\begin{array}{ll}---- \\
\end{array}$ & $6.4-7.2(\mathrm{~m})(9 \mathrm{H})$ \\
\hline A7 & $2.0(\mathrm{t})(2 \mathrm{H})$ & $2.1(\mathrm{t})(2 \mathrm{H})$ & $6.0(\mathrm{~S})(1 \mathrm{H})$ & $2.9(\mathrm{~S})(1 \mathrm{H})$ & $6.3-7.1(\mathrm{~m})(8 \mathrm{H})$ \\
\hline A8 & $2.0(\mathrm{t})(2 \mathrm{H})$ & $2.2(\mathrm{t})(2 \mathrm{H})$ & $6.3(\mathrm{~S})(1 \mathrm{H})$ & ----- & $6.3-7.2(\mathrm{~m})(8 \mathrm{H})$ \\
\hline
\end{tabular}

The ${ }^{1} \mathrm{H}-\mathrm{NMR}$ spectra of compound $\mathrm{B} 1$ is taken as a representative model for the discussion, which showed a singlet signal at $\delta 4.6 \mathrm{ppm}$ related to methylene protons $\left(\mathrm{CH}_{2}\right)$ $\left(\mathrm{C}_{1}\right)$, another singlet signal at $\delta 7.2 \mathrm{ppm}$ related to $(\mathrm{CH})$ benzyl proton $(=\mathrm{CH})$, (Table 7).

The (FT-IR) showed absorption bands in the range of (1606-1594) $\mathrm{cm}^{-1}$ related to the stretching vibration of the $(\mathrm{C}=\mathrm{N})$ bond, (Table4).

Table 7: ${ }^{1} \mathrm{H}-\mathrm{NMR}$ of (some Schiff bases)

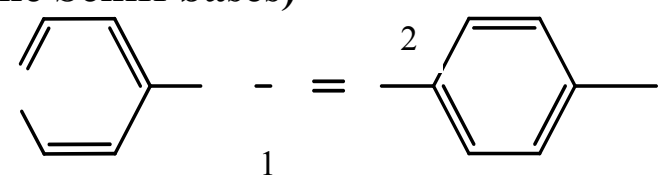

\begin{tabular}{|c|c|c|c|c|}
\hline \multirow{2}{*}{$\begin{array}{c}\text { Compd. } \\
\text { NO. }\end{array}$} & $\begin{array}{c}{ }^{1} \mathbf{H}-\mathbf{N M R}\left(\mathbf{C D C l}_{3}\right) \mathbf{\delta p p m} \\
\text { methylene } \\
\text { group }\left(\mathbf{C}_{1}-\underline{\underline{H}}\right)\end{array}$ & $\left.\mathbf{( C}_{2}-\underline{\mathbf{H}}\right)$ & others & Ar- $\underline{\mathbf{H}}$ \\
\hline $\mathrm{B} 1$ & $4.6(\mathrm{~S})(2 \mathrm{H})$ & $7.2(\mathrm{~S})(1 \mathrm{H})$ & $\ldots-$ & $7.3-8.4(\mathrm{~m})(9 \mathrm{H})$ \\
\hline $\mathrm{B} 2$ & $3.7(\mathrm{~S})(2 \mathrm{H})$ & $7.4(\mathrm{~S})(1 \mathrm{H})$ & $\begin{array}{c}\mathrm{O}-\mathrm{CH}_{3} \\
4.8(\mathrm{~S})(3 \mathrm{H})\end{array}$ & $7.5-8.8(\mathrm{~m})(8 \mathrm{H})$ \\
\hline $\mathrm{B} 3$ & $4.7(\mathrm{~S})(2 \mathrm{H})$ & $7.5(\mathrm{~S})(1 \mathrm{H})$ & ----- & $7.7-8.4(\mathrm{~m})(8 \mathrm{H})$ \\
\hline B6 & $4.6(\mathrm{~S})(2 \mathrm{H})$ & $7.2(\mathrm{~S})(1 \mathrm{H})$ & $\begin{array}{c}\mathrm{CH}_{3} \\
2.3(\mathrm{~S})(3 \mathrm{H})\end{array}$ & $7.7-8.4(\mathrm{~m})(8 \mathrm{H})$ \\
\hline
\end{tabular}

The Schiff base[N-Arylidene-4-picolylamine] (B1-6) were reacted with chalcones (FTs) 2- (2-furfurylidene-1-tetralone) (A1) under basic conditions to afford the corresponding substituted spiropyrrolidenes (S1-3), also Schiff base [N-Benzylidene-4picolylamine] (B1) and its substituted analogue [N- Arylidene-4-picolylamine] (B2-6) were reacted with 2-benzylidine-1-tetralone (A6) and substituted 2-benzylidene-1-tetralone (A7, A8) under basic conditions to afford the corresponding substituted spiro- pyrrolidenes (S4-15). 
The ${ }^{1} \mathrm{H}-\mathrm{NMR}$ spectrum of compound S1 and S4 are taken as representative models for the discussion. shifts:

The Spectrum of compound (S1) showed significant bands at the following chemical

A broad singlet signal at $\delta 2.5 \mathrm{ppm}$ related to N-H proton, a singlet signal at $\delta 4.7$ ppm to $\mathrm{C}_{2}$, protons a doublet signal at $\delta 3.8 \mathrm{ppm}$ due to $\mathrm{C}_{4}$ protons , another doublet signal at $\delta 4.4 \mathrm{ppm}$ due to of $\mathrm{C}_{5}$ protons.

The aromatic protons $(16 \mathrm{H})$ resonate at the range of $\delta(6.2-8.1) \mathrm{ppm}$. Spectrum of compound (S4) showed significant bands at the following chemical shifts:

A singlet signal at $\delta 2.3 \mathrm{ppm}$ related to $\mathrm{N}-\mathrm{H}$ proton. a singlet signal at $\delta 4.6 \mathrm{ppm}$ related to $\mathrm{C}_{2}$ protons, a doublet signal at $\delta 3.6 \mathrm{ppm}$ due $\mathrm{C}_{4}$ protons, and a doublet signal at $\delta 4.4$ ppm due to of $\mathrm{C}_{5}$ protons.

The aromatic protons $(18 \mathrm{H})$ resonate within the range of $\delta(7.0-8.0) \mathrm{ppm}$. The ${ }^{1} \mathrm{H}-\mathrm{NMR}$ spectra for the other synthesized pyrrolidines are listed in Table (8).

The (FT-IR) spectral (Table 9) exhibit the main absorption bands for compounds (S1$15)$.

A bands at the range of (1654-1715) $\mathrm{cm}^{-1}$, related to the stretching vibration of the carbonyl group. a band at $3393-3446 \mathrm{~cm}^{-1}$ corresponds to the stretching vibration of N-H. In the case of the compounds (S1, S2, S3, S6, S7, S11, S12, S14), the absorption bands appeared at the range of (1218-1292) $\mathrm{cm}^{-1}$ related to the bonds of C-O-C, but in case of compounds (S3, S8, S12, S13) the nitro groups, showed absorption bands in the range of (1313-1560) $\mathrm{cm}^{-1}$ due to the symmetric and asymmetric stretching $\mathrm{N}=\mathrm{O}$ respectively (Table 9). Also in the case of compounds (S5, S9, S10, S13), the band at (741-772) $\mathrm{cm}^{-1}$ is related to the $\mathrm{C}-\mathrm{Cl}$ stretching vibration.

The UV spectra (Field et al., 2007) (Mistry, 2009) (Table 9) show a maximum absorption of compounds $\mathrm{S}(1-15)$ in the range of (266-352)nm, which indicates blue shift with respect to $\lambda_{\max }$ of the Chalcones (ATs and FTs). 
Table 8: ${ }^{1} \mathrm{H}-\mathrm{NMR}$ spectral data of some spiropyrrolidine compounds

\begin{tabular}{|c|c|c|c|c|c|c|c|c|}
\hline \multirow{2}{*}{$\begin{array}{c}\text { Compd. } \\
\text { No. }\end{array}$} & \multicolumn{8}{|c|}{${ }^{1} \mathrm{H}-\mathrm{NMR}\left(\mathrm{CDCl}_{3}\right) \delta \mathrm{ppm}$} \\
\hline & $\mathrm{C}_{6-}-\mathrm{H}$ & $\mathrm{C}_{7}-\mathbf{H}$ & $\mathrm{C}_{4}-\underline{\mathrm{H}}$ & $\mathrm{OCH}_{3}$ & $\mathrm{C}_{5}-\underline{\mathrm{H}}$ & $\mathrm{C}_{2}-\underline{\mathrm{H}}$ & Ar-H & N-H \\
\hline S1 & $\begin{array}{l}2.3(\mathrm{t}) \\
(2 \mathrm{H})\end{array}$ & $\begin{array}{l}2.4(\mathrm{t}) \\
(2 \mathrm{H})\end{array}$ & $\begin{array}{c}3.8(\mathrm{~d}) \\
1 \mathrm{H}\end{array}$ & --- & $\begin{array}{c}4.4(\mathrm{~d}) \\
1 \mathrm{H}\end{array}$ & $\begin{array}{c}4.7(\mathrm{~S}) \\
1 \mathrm{H}\end{array}$ & $\begin{array}{c}6.2-8.1(\mathrm{~m}) \\
16 \mathrm{H}\end{array}$ & $\begin{array}{c}8.5(\mathrm{~S}) \\
1 \mathrm{H}\end{array}$ \\
\hline S4 & $\begin{array}{r}2.2(\mathrm{t}) \\
(2 \mathrm{H}) \\
\end{array}$ & $\begin{array}{l}2.3(\mathrm{t}) \\
(2 \mathrm{H})\end{array}$ & $\begin{array}{c}3.6(\mathrm{~d}) \\
1 \mathrm{H}\end{array}$ & ---- & $\begin{array}{c}4.4(\mathrm{~d}) \\
1 \mathrm{H}\end{array}$ & $\begin{array}{c}4.6(\mathrm{~S}) \\
1 \mathrm{H}\end{array}$ & $\begin{array}{c}7.0-8.0(\mathrm{~m}) \\
18 \mathrm{H} \\
\end{array}$ & $\begin{array}{c}8.4(\mathrm{~S}) \\
1 \mathrm{H} \\
\end{array}$ \\
\hline S5 & $\begin{array}{l}2.3(\mathrm{t}) \\
(2 \mathrm{H})\end{array}$ & $\begin{array}{l}2.4(\mathrm{t}) \\
(2 \mathrm{H})\end{array}$ & $\begin{array}{c}3.7(\mathrm{~d}) \\
1 \mathrm{H}\end{array}$ & ---- & $\begin{array}{c}4.3(\mathrm{~d}) \\
1 \mathrm{H}\end{array}$ & $\begin{array}{c}4.6(\mathrm{~S}) \\
1 \mathrm{H} \\
\end{array}$ & $\begin{array}{c}7.0-7.9(\mathrm{~m}) \\
17 \mathrm{H} \\
\end{array}$ & $\begin{array}{c}8.4(\mathrm{~S}) \\
1 \mathrm{H} \\
\end{array}$ \\
\hline S7 & $\begin{array}{l}2.3(\mathrm{t}) \\
(2 \mathrm{H})\end{array}$ & $2.4(\mathrm{t})(2 \mathrm{H})$ & $\begin{array}{c}3.6(\mathrm{~d}) \\
1 \mathrm{H}\end{array}$ & $\begin{array}{c}3.5(\mathrm{~S}) \\
3 \mathrm{H}\end{array}$ & $\begin{array}{c}4.2(\mathrm{~d}) \\
1 \mathrm{H}\end{array}$ & $\begin{array}{c}4.7(\mathrm{~S}) \\
1 \mathrm{H}\end{array}$ & $\begin{array}{c}6.9-7.8(\mathrm{~m}) \\
17 \mathrm{H}\end{array}$ & $\begin{array}{c}8.4(\mathrm{~S}) \\
1 \mathrm{H}\end{array}$ \\
\hline S8 & $\begin{array}{l}2.1(\mathrm{t}) \\
(2 \mathrm{H})\end{array}$ & $\begin{array}{l}2.3(\mathrm{t}) \\
(2 \mathrm{H})\end{array}$ & $\begin{array}{c}3.7(\mathrm{~d}) \\
1 \mathrm{H}\end{array}$ & ---- & $\begin{array}{c}4.7(\mathrm{~d}) \\
1 \mathrm{H}\end{array}$ & $\begin{array}{c}4.8(\mathrm{~S}) \\
1 \mathrm{H}\end{array}$ & $\begin{array}{c}7.0-7.8(\mathrm{~m}) \\
17 \mathrm{H}\end{array}$ & $\begin{array}{c}8.6(\mathrm{~S}) \\
1 \mathrm{H}\end{array}$ \\
\hline $\mathrm{S} 10$ & $\begin{array}{l}2.3(\mathrm{t}) \\
(2 \mathrm{H})\end{array}$ & $\begin{array}{l}2.4 \mathrm{t} \\
(2 \mathrm{H})\end{array}$ & $\begin{array}{c}3.6(\mathrm{~d}) \\
1 \mathrm{H}\end{array}$ & --- & $\begin{array}{c}4.4(\mathrm{~d}) \\
1 \mathrm{H}\end{array}$ & $\begin{array}{c}4.7(\mathrm{~S}) \\
1 \mathrm{H}\end{array}$ & $\begin{array}{c}7.0-7.6(\mathrm{~m}) \\
17 \mathrm{H}\end{array}$ & $\begin{array}{c}8.3(\mathrm{~S}) \\
1 \mathrm{H}\end{array}$ \\
\hline S11 & $\begin{array}{l}2.1(\mathrm{t}) \\
(2 \mathrm{H})\end{array}$ & $\begin{array}{l}2.3(\mathrm{t}) \\
(2 \mathrm{H})\end{array}$ & $\begin{array}{c}3.7(\mathrm{~d}) \\
1 \mathrm{H}\end{array}$ & $\begin{array}{c}3.9(\mathrm{~S}) \\
3 \mathrm{H}\end{array}$ & $\begin{array}{c}4.6(\mathrm{~d}) \\
1 \mathrm{H}\end{array}$ & $\begin{array}{c}4.8(\mathrm{~S}) \\
1 \mathrm{H}\end{array}$ & $\begin{array}{c}6.7-8.0(\mathrm{~m}) \\
16 \mathrm{H} \\
\end{array}$ & $\begin{array}{c}8.6(\mathrm{~S}) \\
1 \mathrm{H} \\
\end{array}$ \\
\hline S12 & $\begin{array}{l}2.3(\mathrm{t}) \\
(2 \mathrm{H})\end{array}$ & $\begin{array}{l}2.4(\mathrm{t}) \\
(2 \mathrm{H})\end{array}$ & $\begin{array}{c}3.4(\mathrm{~d}) \\
1 \mathrm{H}\end{array}$ & $\begin{array}{c}3.7(\mathrm{~S}) \\
3 \mathrm{H}\end{array}$ & $\begin{array}{c}3.9(\mathrm{~d}) \\
1 \mathrm{H}\end{array}$ & $\begin{array}{c}4.7(\mathrm{~S}) \\
1 \mathrm{H}\end{array}$ & $\begin{array}{c}6.7-8.1(\mathrm{~m}) \\
16 \mathrm{H}\end{array}$ & $\begin{array}{c}8.4(\mathrm{~S}) \\
1 \mathrm{H}\end{array}$ \\
\hline $\mathrm{S} 13$ & $\begin{array}{l}2.1(\mathrm{t}) \\
(2 \mathrm{H})\end{array}$ & $\begin{array}{l}2.4(\mathrm{t}) \\
(2 \mathrm{H})\end{array}$ & $\begin{array}{c}3.7(\mathrm{~d}) \\
1 \mathrm{H}\end{array}$ & ---- & $\begin{array}{c}4.3(\mathrm{~d}) \\
1 \mathrm{H}\end{array}$ & $\begin{array}{c}4.6(\mathrm{~S}) \\
1 \mathrm{H}\end{array}$ & $\begin{array}{c}7.0-8.0(\mathrm{~m}) \\
16 \mathrm{H} \\
\end{array}$ & $\begin{array}{c}8.4(\mathrm{~S}) \\
1 \mathrm{H} \\
\end{array}$ \\
\hline S14 & $\begin{array}{l}2.1(\mathrm{t}) \\
(2 \mathrm{H})\end{array}$ & $\begin{array}{l}2.2(\mathrm{t}) \\
(2 \mathrm{H})\end{array}$ & $\begin{array}{c}3.7(\mathrm{~d}) \\
1 \mathrm{H}\end{array}$ & $\begin{array}{c}3.89 \mathrm{~S}) \\
3 \mathrm{H}\end{array}$ & $\begin{array}{c}4.5(\mathrm{~d}) \\
1 \mathrm{H}\end{array}$ & $\begin{array}{c}4.6(\mathrm{~S}) \\
1 \mathrm{H}\end{array}$ & $\begin{array}{c}7.0-8.1(\mathrm{~m}) \\
16 \mathrm{H} \\
\end{array}$ & $\begin{array}{c}8.2(\mathrm{~S}) \\
1 \mathrm{H} \\
\end{array}$ \\
\hline S15 & $\begin{array}{l}2.2(\mathrm{t}) \\
(2 \mathrm{H})\end{array}$ & $\begin{array}{l}2.3(\mathrm{t}) \\
(2 \mathrm{H})\end{array}$ & $\begin{array}{c}3.6(\mathrm{~d}) \\
1 \mathrm{H}\end{array}$ & $\begin{array}{c}\mathrm{CH}_{3} \\
2.6(\mathrm{~S}) \\
3 \mathrm{H}\end{array}$ & $\begin{array}{c}4.5(\mathrm{~d}) \\
1 \mathrm{H}\end{array}$ & $\begin{array}{c}4.7(\mathrm{~S}) \\
1 \mathrm{H}\end{array}$ & $\begin{array}{c}7.0-8.1(\mathrm{~m}) \\
17 \mathrm{H}\end{array}$ & $\begin{array}{c}8.2(\mathrm{~S}) \\
1 \mathrm{H}\end{array}$ \\
\hline
\end{tabular}


Table 9: U.V and IR Spectral data of pyrrolidines (S1-15).

\begin{tabular}{|c|c|c|c|c|c|}
\hline \multirow[b]{2}{*}{$\begin{array}{c}\text { Compd. } \\
\text { No. }\end{array}$} & \multicolumn{5}{|c|}{ IR $(\mathrm{KBr}), \mathrm{v}\left(\mathrm{cm}^{-1}\right)$} \\
\hline & $\mathbf{C}=\mathbf{O}$ & $\mathrm{C}=\mathrm{C}$ & N-H & Others & $\begin{array}{c}\text { U.V } \\
\lambda_{\max }(\mathbf{n m})\end{array}$ \\
\hline S1 & 1681 & 1530 & 3415 & C-O-C 1232 & 270,328 \\
\hline S2 & 1654 & 1592 & 3446 & C-O-C 1250 & 340 \\
\hline S3 & 1652 & 1594 & 3393 & $\begin{array}{c}\mathrm{C}-\mathrm{O}-\mathrm{C} 1233 \\
\mathrm{~N}=\mathrm{O} \text { sym } 1313 \\
\text { asym } 1560\end{array}$ & 352 \\
\hline S4 & 1715 & 1597 & 3432 & ---- & 282 \\
\hline S5 & 1656 & 1598 & 3412 & C-Cl 741 & 270 \\
\hline S6 & 1661 & 1598 & 3414 & C-O-C 1218 & 292 \\
\hline S7 & 1657 & 1598 & 3413 & C-O-C 1231 & 270 \\
\hline S8 & 1666 & 1579 & 3418 & $\begin{array}{c}\mathrm{N}=\mathrm{O} \text { sym } 1345 \\
\text { asym } 1518\end{array}$ & 282 \\
\hline S9 & 1650 & 1576 & 3438 & C-Cl 772 & 266 \\
\hline $\mathrm{S} 10$ & 1681 & 1601 & 3452 & $\mathrm{C}-\mathrm{Cl} 747$ & 268 \\
\hline $\mathrm{S} 11$ & 1666 & 1590 & 3430 & C-O-C 1230 & 274 \\
\hline $\mathrm{S} 12$ & 1665 & 1592 & 3439 & $\begin{array}{c}\mathrm{C}-\mathrm{O}-\mathrm{C} 1219 \\
\mathrm{~N}=\mathrm{O} \text { sym } 1324 \\
\text { asym } 1543\end{array}$ & 270,318 \\
\hline $\mathrm{S} 13$ & 1665 & 1597 & 3480 & $\begin{array}{c}\mathrm{N}=\mathrm{O} \text { sym } 1334 \\
\text { asym } 1494 \\
\mathrm{C}-\mathrm{Cl} 752\end{array}$ & 286 \\
\hline S14 & 1686 & 1628 & 3415 & C-O-C 1258 & 292,350 \\
\hline $\mathrm{S} 15$ & 1697 & 1598 & 3427 & $\begin{array}{ll}---- \\
\end{array}$ & 268,402 \\
\hline
\end{tabular}

REFERENCES

Bin, L.; Xi-Qun, L.; Wen-Jieomed, Z. ; Me-Yun, Z. (2009). Synthesis of ionic liquidsupported Schiff bases , ARKIVOC, Xi, 165- 171.

Deshong, P.; Leginus, M. (1983). Stereospecific synthesis of racemic daunosamine. Diastereofacial selectivity in a nitrone cycloaddition. J. Am. Chem. Soc., 105, 1686.

Fisera, L.; Sauter, F.; Frolich, J.; Feng, Y.; Ertl, P.; Mereiter, K., (1994). Synthesis of spirosubstituted 1,3oxazines by a new sequence leading to spiroheterocycles. Monatsh. Chem. , 125, 909.

Field, L.D. ; Sternhen, S. ; Kalman, R. (2007). "Organic Structures from Spectra", 4th edn. John-Wiley and Sons. New York, pp.30-34.

Ghandi, M.; Yari, A.; Jamal, S. ; Taheri, A. (2009). Synthesis of novel spiropyrrolidine through 1,3-dipolar cycloaddition, Tetrahedron Letteres, 50, 4724-472.

Jayashankaran, J.; Durga, R. ; Raghunathan, R., (2005). A facile entry into a novel class of dispiroheterocycles through 1,3-dipolar cycloaddition. ARKIVOC, 32-39.

Katritzky, R.; Ress, C.W. (1997). Comprehensive heterocyclic chemistry. Elsevier Sci. Ltd., 256-260. 
Luibineau, A.; Bouchain, G.; Queneau, Y. (1995). Pyrrolidine and 1,3-oxazolidine formation from azomethine ylides influenced by change from classical conditions to microwave irradiation. J. Chem. Soc, Perkin Trans I, 2433.

Manikandan, S.; Mohamed Ashraf, M.; Raghunathan, R. (2001). A formal [3 2] cycloaddition strategy for the synthesis of unique class of dispiroheterocycles, Synth. Commun., 31, 3593.

March, J.A. (1992). "Advanced Organic Chemistry". 4th edn., Mc Graw-Hill, Inc., New York, $896 \mathrm{p}$.

Mistry, B.D. (2009). A Handbook of Spectroscopic Data". Oxford book company.

Parich, V. M. (1974). "Absorption Spectroscopy of Organic Compounds". AddisionWesley publishing company, Inc., pp. 247-252, pp. 51- 59, pp. 60-64.

Popandova-Yambolieva, K. ; Ivanov, C. (1989). Synthesis of new spiropyrrolidines and Michael addition products using phase transfer catalyzed addition of schiff bases to 9-arylmethylenefluorenes. Chemica. Scripta. 29, 269-271.

Poornachandran, M. ; Raghunathan, R. (2010). A novel access to dispirocyclohexanoneindan pyrrolidine, Indian J. Chemistry, 49B, 127-130.

Raj, A. A. ; Raghnathan, R. (2003). Synthesis of spiropyrrolidine via formal [3,2] cycloaddition of unusual enones and cis 3-benzoy 1-1-cyclohexy 1-2phenylaziridine. Tetrahedron, 59, 2907-2911.

Waldmann, H. (1995). "Organic Synthesis in Water". Thamson Science, Pappellallee, Weinheim, Germany, Synlett, 133p.

Shriner, R. L. ; Fuson, R.C. ; Curtin, D.Y., (1964). "The Systematic Identification of Organic Compounds". 5th edn., John Wiley and Sons Inc., New York. 29p.

Subramaniyan, G.; Raghunathan, R. (2001). Synthesis of highly substituted spiropyrrolidines via 1,3-dipolar cycloaddition reaction of $\mathrm{N}$-metalated azomethine ylides. Tetrahedron, 57, 2909.

Vogel, A. (1989). "Practical Organic Chemistry". Longmans, 5th edn. 1034 p. 